\title{
Controllable enhanced dragging of light in ultradispersive media
}

\author{
Sankar Davuluri and Yuri V. Rostovtsev \\ Department of Physics, University of North Texas, Denton, Texas 76203, USA
}

(Received 25 March 2012; published 5 July 2012)

\begin{abstract}
We have theoretically demonstrated an enhanced Fizeau effect due to dragging the light that occurs when the group velocity of light is ultraslow. The proposed experiment can be done in a cell of atomic $\mathrm{Rb}$ vapor under conditions such that the group velocity of light is of the order of a few hundred meters per second. We show theoretically that higher-order dispersion can influence the Fizeau effect and can be observed experimentally. It has been shown that the change of phase is sensitive to the motion of the cell with the speed of the order of $10^{-3} \mathrm{~cm} / \mathrm{s}$ and for possible displacements as small as $10 \AA$. The enhanced dragging effect can be applied for position control, detection of slow mechanical motion, and efficient modulators of light.
\end{abstract}

DOI: 10.1103/PhysRevA.86.013806

PACS number(s): 42.50.Gy, 42.50.Wk, 42.50.Nn, 42.25.Bs

\section{INTRODUCTION}

The fact that the speed of light in a moving medium differs from its value in a stationary medium has been known for over a century [1-3]. In a medium moving with velocity $v$ (see Fig. 1), the phase velocity of light is given by

$$
u=u_{0}+v F
$$

where $u_{0}=c / n$ is the phase velocity of light with respect to the medium, $n$ is the index of refraction of the medium, and $F$ is the Fresnel drag coefficient. Usually, in gases $n \simeq 1$, the Fresnel drag coefficient $F$ is negligible.

Quantum coherence effects, such as coherent population trapping (CPT) [4] and electromagnetically induced transparency (EIT) [5-8], have been the focus of broad research activity for the last two decades, as they are able to drastically change the optical properties of media. For example, for EIT in $\mathrm{CW}$ and pulsed regimes [6-10], absorption practically vanishes. The medium with excited quantum coherence, phaseonium [5], can be used to make an ultradispersive prism [11] that has several orders of magnitude higher angular spectral dispersion. The bending of light has been also demonstrated using the transverse dragging effect [12]. The corresponding steep dispersion results in the ultraslow or ultrafast propagation of light pulses [13-17], which can produce huge optical delays [17] and can be used for drastic modification of the phase-matching conditions for Brillouin scattering [18], four-wave mixing [19], controllable switching between bunching and antibunching [20], storage and retrieval of pulses [21,22], freezing of a light pulse [23], and ultrahigh enhancement in absolute and relative rotation sensing using fast and slow light [24].

In this paper, we study the dragging of light by a gas medium, and we predict a nonlinear dependence of the Fizeau effect on the velocity of a cell that can be observed experimentally. The dragging effect in ultradispersive media can be enhanced and can be controlled by its strong dependence on the parameters of the driving beam, such as the Rabi frequency and the detuning. It is shown below that the change of phase is sensitive to the motion of the cell with a velocity of the order of $10^{-3} \mathrm{~cm} / \mathrm{s}$ and for possible displacements as small as $10 \AA$. Applications of the enhanced dragging effect can be found for efficient modulators of light, position control, and detection of slow motion.

\section{MOVING DIELECTRICS}

Consider an electromagnetic wave propagating with wave number $k_{0}$ and frequency $\omega_{0}$ in a dielectric medium. The dispersion relation between $k_{0}$ and $\omega_{0}$ is given by

$$
k_{0}=\frac{\omega_{0}}{c} n\left(\omega_{0}\right) \text {, }
$$

where $n\left(\omega_{0}\right)$ is the index of refraction of the medium. Let us assume the medium moves with velocity $v(v \ll c, c$ is the velocity of the light in vacuum) with respect to the laboratory frame. The wave number and frequency of the wave are $k$ and $\omega$, respectively, and the transformation from the laboratory to the frame related to the medium is given by

$$
\omega_{0}=\omega-v k, \quad k_{0}=k-\frac{v}{c^{2}} \omega .
$$

Then, the dispersion relation in the laboratory frame is given by

$$
k-\frac{v}{c^{2}} \omega=\frac{\omega-v k}{c} n(\omega-v k) .
$$

Assuming that the velocity is very small, we can use series with respect $k v$ to the second order as

$$
k-\frac{v}{c^{2}} \omega=\frac{\omega}{c} n(\omega)-v k \frac{\partial}{\partial \omega}\left(\frac{\omega}{c} n(\omega)\right)+\frac{v^{2} k^{2}}{2 c} \frac{\partial^{2} \omega n(\omega)}{\partial \omega^{2}},
$$

and the phase velocity can be written as

$$
\frac{\omega}{k}=\frac{c}{n}+v\left(\frac{n_{g}}{n}-\frac{1}{n^{2}}+\frac{v \omega V_{g}^{\prime}}{2 V_{g}^{2}}-\frac{v}{n^{2} V_{g}}+\frac{v}{n^{3} c}\right),
$$

where $V_{g}^{\prime}=\partial V_{g} / \partial \omega$, and $F$, the Fresnel drag coefficient $[2,3]$ [see also Eq. (1)], is given by

$$
F=1-\frac{1}{n^{2}}+\frac{\omega}{n} \frac{\partial n(\omega)}{\partial \omega} \equiv \frac{n_{g}}{n}-\frac{1}{n^{2}},
$$

where $n$ is the index of refraction of the medium, $n_{g}=c / V_{g}=$ $n+\omega \frac{\partial n(\omega)}{\partial \omega}$ is the group index, and $V_{g}$ is the group velocity.

Then, the wave vector is given by

$$
k=\frac{\frac{\omega}{c}\left(n+\frac{v}{c}\right)+\frac{1}{2} \frac{2 n^{\prime}+\omega n^{\prime \prime}}{c} \frac{v^{2} \omega^{2}\left(n+\frac{v}{c}\right)^{2}}{\left[c+v\left(n+\omega n^{\prime}\right)\right]^{2}}}{1+\frac{v}{c}\left(n+\omega n^{\prime}\right)},
$$




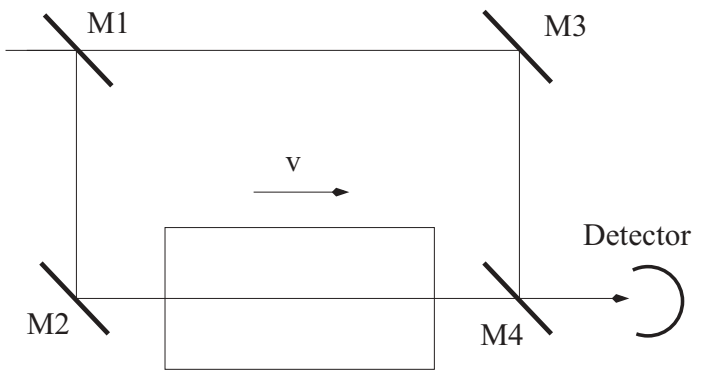

FIG. 1. Interferometer to measure the Fizeau effect. The light beam is split into two beams: one goes through a moving medium and the other through free space. $\mathrm{M}_{1}, \mathrm{M}_{2}, \mathrm{M}_{3}$, and $\mathrm{M}_{4}$ are the mirrors. Two beams combined together on mirror $\mathrm{M}_{4}$, and the signal of the detector depends on the interference between combined beams.

where $n^{\prime}=\frac{\partial n(\omega)}{\partial \omega}$ and $n^{\prime \prime}=\frac{\partial^{2} n(\omega)}{\partial \omega^{2}}$, and the phase shift up to the second order on velocity $v$ is given by

$$
\Phi=\left(k-\frac{\omega}{c} n\right) L \simeq \frac{\omega L}{c}\left(\frac{2 \omega n^{\prime}+\omega^{2} n^{\prime \prime}}{2} \frac{v^{2}}{c^{2}}-\frac{v}{V_{g}}\right) .
$$

\section{MODEL}

Now, let us consider a dielectric medium consisting of $\Lambda$ type atoms. A strong field of frequency $\omega_{d}$ is the coupling field, and a weak field of frequency $\omega_{p}$ is the probe field. The probe and coupling fields can be represented as

$$
E_{p, d}=E_{p, d}^{0} \exp \left[i k_{p, d} z-i \omega_{p, d} t\right],
$$

where $E_{p, d}^{0}$ is the amplitude of the fields at $z=0$.

The interaction Hamiltonian in the rotating-wave approximation can be written as

$$
H=\hbar\left[\Omega_{p}^{*} e^{i \Delta_{p} t}|b\rangle\left\langle a\left|+\Omega^{*} e^{i \Delta_{d} t}\right| c\right\rangle\langle a|+\operatorname{adj}\right]
$$

where $|b\rangle\langle a|$ and $|c\rangle\langle a|$ are the atomic projection operators, $\Omega_{p}=\wp_{a b} E_{p}^{0} / \hbar$ and $\Omega=\wp_{a c} E_{d}^{0} / \hbar$ are the probe and coupling Rabi frequencies, $\Delta_{p}=\omega_{a b}-\omega_{p}$ and $\Delta_{d}=\omega_{a c}-\omega_{d}$ are detunings for probe and coupling laser beams, and $\wp_{a b}$ and $\wp_{a c}$ are the dipole momenta of the transitions. The coupling field is resonant with the $|a\rangle \rightarrow|c\rangle$ transition $\left(\Delta_{d}=0\right)$.

The density matrix equations are given by

$$
\frac{\partial \rho}{\partial t}=\frac{i}{\hbar}[\rho, H]-\frac{1}{2}(\Gamma \rho+\rho \Gamma),
$$

where $\Gamma$ is the matrix of relaxation rates for all components of the density matrix $\rho$. The temporal and spatial evolution of fields is determined by the propagation equations,

$$
\frac{\partial^{2} E_{p, d}}{\partial z^{2}}-\frac{1}{c^{2}} \frac{\partial^{2} E_{p, d}}{\partial t^{2}}=\frac{4 \pi}{c^{2}} \frac{\partial^{2} P_{p, d}}{\partial t^{2}},
$$

which in a steady-state and slowly varying amplitude approximation can be written as

$$
\frac{\partial \Omega_{p}}{\partial z}=-i \frac{2 \pi \omega}{c} P_{a b}, \quad \frac{\partial \Omega}{\partial z}=-i \frac{2 \pi \omega}{c} P_{a c},
$$

where $P_{a b}$ and $P_{a c}$ are the atomic polarizations at the corresponding drive and probe transitions. Solving Eq. (11) in a steady-state approximation, the atomic polarization at the probe frequency can be found as

$$
P_{a b}=N \wp_{a b} \rho_{a b}=\frac{\epsilon-1}{4 \pi} E_{p}=\frac{-i N \wp_{a b} \Omega_{p}}{\Gamma_{a b}+\frac{|\Omega|^{2}}{\Gamma_{c b}}},
$$

where $\quad \Gamma_{c b}=\gamma_{c b}+i\left(\omega_{c b}-\omega_{p}+\omega_{d}\right) \quad$ and $\quad \Gamma_{a b}=\gamma_{a b}+$ $i\left(\omega_{a b}-\omega_{p}\right)$. The dielectric function is given by

$$
\epsilon=1-i \frac{4 \pi\left|\wp_{a b}\right|^{2} N}{\hbar} \frac{\Gamma_{c b}}{\Gamma_{a b} \Gamma_{c b}+|\Omega|^{2}},
$$

and the index of refraction is given by

$$
n=1-i \frac{\eta \gamma_{r} \Gamma_{c b}}{\Gamma_{a b} \Gamma_{c b}+|\Omega|^{2}},
$$

where $\eta=\frac{3 \lambda^{3} N}{16 \pi^{2}}$ and $\gamma_{r}=4 \omega_{a b}^{3}\left|\wp_{a b}\right|^{2} / 3 \hbar c^{3}$. The Doppler broadening is important to take into account; it has been taken into account by averaging the index of refraction over the velocity distribution [25] (see also the Appendix):

$$
n_{D}=\int_{-\infty}^{+\infty} d v f(u) n\left(\omega_{p}, u\right)
$$

where $f(u)$ is the velocity distribution of the atoms. To simplify calculations, we take into account the Doppler broadening by approximating the distribution function $f(v)$ by a Lorentzian; in this case the integration can be easily done, and the results are very close to the integration of the Gaussian distribution (see Refs. [14,25]). The typical real (solid lines) and imaginary (dashed lines) parts of the index of refraction are shown in Fig. 2 as a function of the probe frequency detuning from the frequency of transition. The Rb parameters are chosen for simulations: $\Delta_{d}=0, \Omega=3.5 \times 10^{5} \mathrm{~s}^{-1}$, $\gamma_{r}=2 \times 10^{7} \mathrm{~s}^{-1}, \gamma_{a b}=\gamma_{r}+\Delta_{D}, \gamma_{c b}=3 \times 10^{3} \mathrm{~s}^{-1}$, and the density of $\mathrm{Rb}$ atoms is $10^{13} \mathrm{~cm}^{-3}$. Simulations perform as well without Doppler broadening $\left(\Delta_{D}=0\right)$ as with taking the Doppler broadening into account $\left(\Delta_{D}=0.991 \times 10^{9} \mathrm{~s}^{-1}\right)$. Plots shown in Fig. 2(a) correspond to the case without Doppler broadening, and plots shown in Fig. 2(b) correspond to when the Doppler broadening is taken into account. For the phase shift, Eq. (9), the derivatives of the index of refraction are

$$
n^{\prime}=-\frac{\eta \gamma_{r}\left(1-\frac{|\Omega|^{2}}{\Gamma_{c b}^{2}}\right)}{\left(\Gamma_{a b}+\frac{|\Omega|^{2}}{\Gamma_{c b}}\right)^{2}}
$$

and

$$
n^{\prime \prime}=-\frac{2 i \eta \gamma_{r}\left(1-\frac{|\Omega|^{2}}{\Gamma_{c b}^{2}}\right)^{2}}{\left(\Gamma_{a b}+\frac{|\Omega|^{2}}{\Gamma_{c b}}\right)^{3}}-\frac{2 i \eta \gamma_{r}|\Omega|^{2}}{\Gamma_{c b}^{3}\left(\Gamma_{a b}+\frac{|\Omega|^{2}}{\Gamma_{c b}}\right)^{2}} .
$$

\section{DISCUSSION OF THE OBTAINED RESULTS AND THEIR APPLICATIONS}

Let us consider a moving cell filled with a gas of three-level $\Lambda$-type atoms placed inside the interferometer as shown in Fig. 1. The dispersion of the medium can be modified and controlled by the drive field; in particular, the group velocity of light can be very low, and the phase shift [given by Eq. (9)] can be very sensitive to the motion of the cell.

In Fig. 3, we plot the phase shift vs the frequency detuning of the probe field. Figure 3(a) corresponds to the 


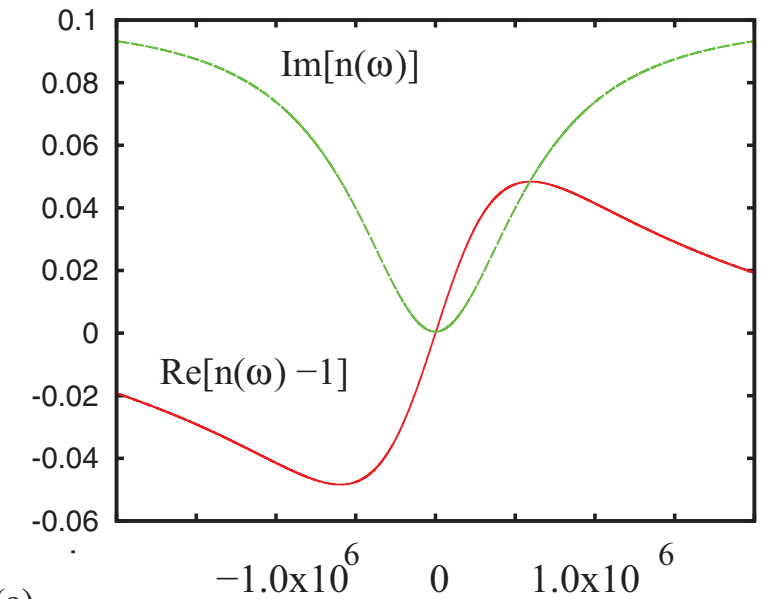

(a)

Probe frequency detuning

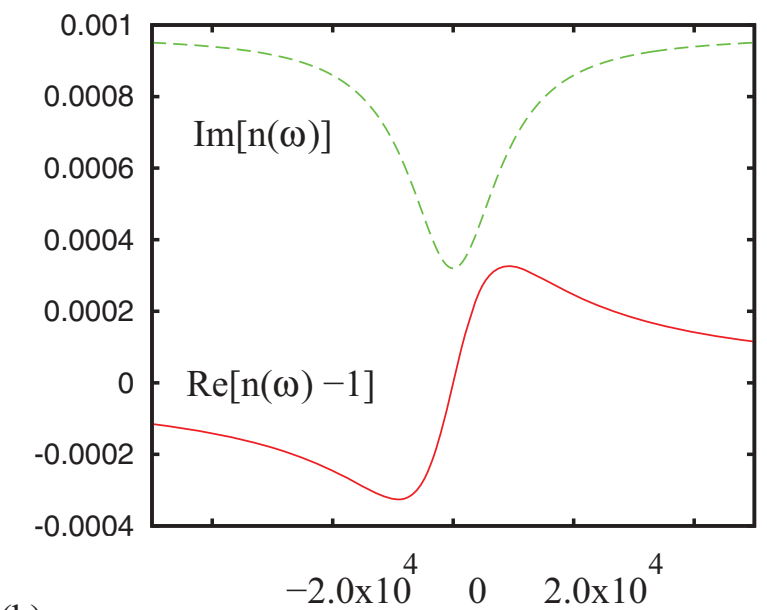

(b)

Probe frequency detuning

FIG. 2. (Color online) Dependence of real (solid line), $\operatorname{Re}[n(\omega)-$ 1], and imaginary (dashed $\operatorname{line}), \operatorname{Im}[n(\omega)]$, parts of the index of refraction of the medium $(n=\operatorname{Re}[n(\omega)]+i \operatorname{Im}[n(\omega)]$ given by Eq. (16)) on the probe frequency detuning (in units of $\mathrm{s}^{-1}$ ) from the frequency of transition, $\omega_{p}-\omega_{a b}$. (a) The Doppler broadening is not taken into account. (b) The Doppler broadening is taken into account.

case without Doppler broadening taken into account, and Fig. 3(b) corresponds to the case when the Doppler broadening is taken into account. The $\mathrm{Rb}$ parameters above are used for simulations, and the velocity of the cell is $10^{-4} \mathrm{~cm} / \mathrm{s}$. One can see that the phase shift is very sensitive to such small velocities. The phase shifts vs the Rabi frequency of the drive field at some detunings of the probe field are shown in Fig. 4. The dependence of the phase shift vs velocity of the cell is shown in Fig. 5. One can clearly see that the phase shift can have a nonlinear dependence on the cell velocity $v$.

It is the steep dispersion of the medium that makes the phase shift very sensitive to the velocity. To understand the results, it is instructive to perform simplified calculations. For this, let us approximate the dispersion only by the linear part that can be seen in Fig. 2. In the frame moving together with the cell, the dispersion relation between wave number $k_{0}$ and frequency $\omega_{0}$

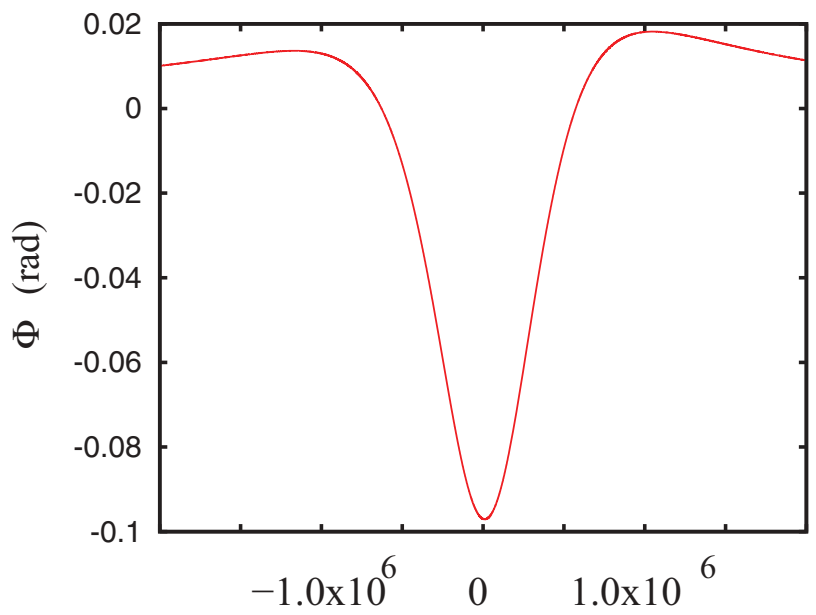

(a)

Probe frequency detuning

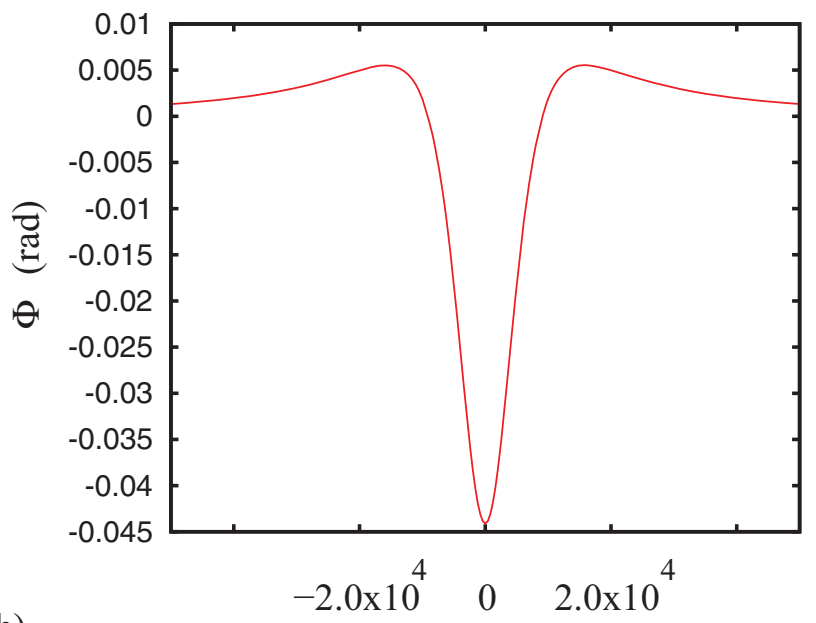

Probe frequency detuning

FIG. 3. (Color online) Dependence of the phase shift $\Phi$ on the probe frequency detuning (in units of $\mathrm{s}^{-1}$ ),$\omega_{p}-\omega_{a b}$, (a) without and (b) with taking into account Doppler broadening.

of light is

$$
k_{0}=\frac{\omega_{a b}}{c}+\frac{\omega_{0}-\omega_{a b}}{V_{g}}=\frac{\omega_{a b}}{c}+\frac{\delta \omega_{0}}{V_{g}} .
$$

Transforming now wave number $k_{0}$ and frequency $\omega_{0}$ of light to wave number $k$ and frequency $\omega$ of light in the laboratory frame,

$$
k=k_{0}+\frac{v}{c^{2}} \omega_{0}, \quad \omega=\omega_{0}+v k_{0}
$$

we obtain

$$
\begin{gathered}
k=\frac{\omega_{a b}}{c}+\frac{\delta \omega_{0}}{V_{g}}+\frac{v}{c^{2}} \omega_{0}=\frac{\omega_{a b}}{c}\left(1+\frac{v}{c}\right)+\delta \omega_{0}\left(\frac{1}{V_{g}}+\frac{v}{c^{2}}\right) \\
\omega=\omega_{a b}\left(1+\frac{v}{c}\right)+\delta \omega_{0}\left(1+\frac{v}{V_{g}}\right),
\end{gathered}
$$




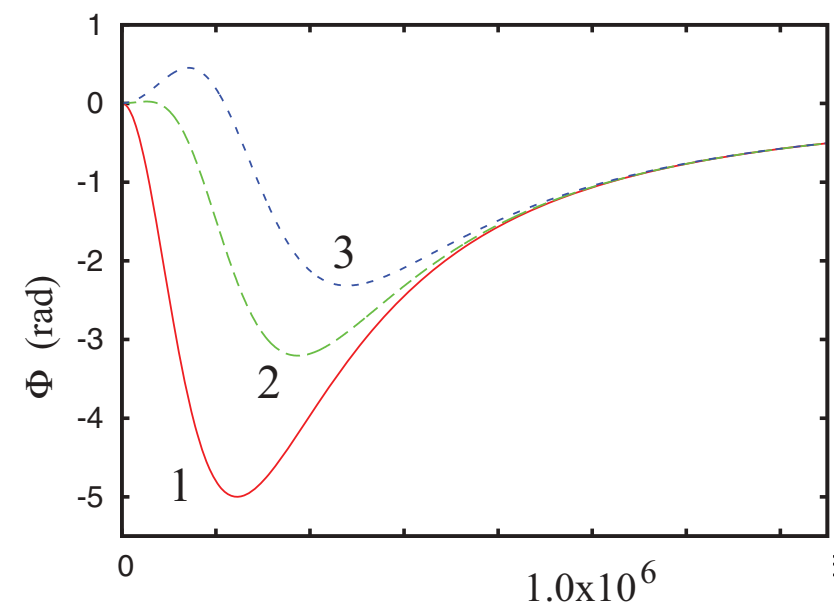

(a)

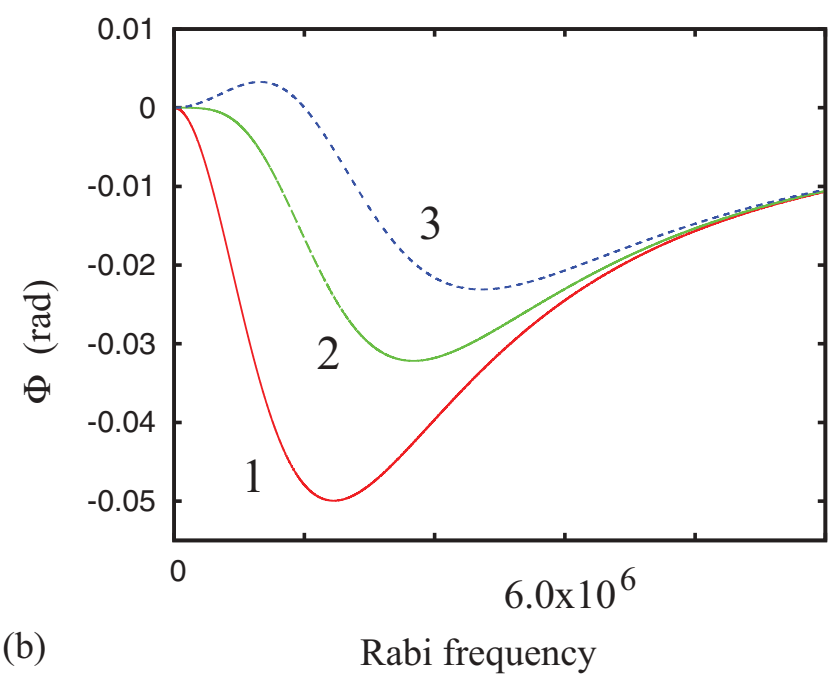

FIG. 4. (Color online) Dependence of the phase shift $\Phi$ on the Rabi frequency (in units of $\mathrm{s}^{-1}$ ). (a) the Doppler broadening is not taken into account. (b) The Doppler broadening is taken into account. The plots are calculated for probe detunings equal to (1) $0 \mathrm{~s}^{-1}$, (2) $3.0 \times 10^{3} \mathrm{~s}^{-1}$, and (3) $5.0 \times 10^{3} \mathrm{~s}^{-1}$.

and, finally,

$$
k=\frac{\omega_{a b}}{c}\left(\frac{V_{g}}{V_{g}+v}+\frac{v}{c}\right)+\frac{\omega-\omega_{a b}}{V_{g}+v}\left(1+\frac{v V_{g}}{c^{2}}\right),
$$

where one can see the modification of the wave number has a $v$ over $V_{g}$ term,

$$
k \simeq \frac{\omega_{a b}}{c}-\frac{\omega_{a b}}{c} \frac{v}{V_{g}} .
$$

Equation (25) leads to a large phase shift of the order

$$
\Phi \simeq-\frac{\omega_{a b}}{c} \frac{L v}{V_{g}}
$$

after propagating a distance $L$ inside the cell [note that it coincides with the linear part of the velocity of Eq. (9)]. For parameters such as $L=1.0 \mathrm{~cm}, \omega_{a b} / c=7.9 \times 10^{4} \mathrm{~cm}^{-1}$, and $V_{g}=10^{4} \mathrm{~cm} / \mathrm{s}$ and assuming the easily detectable minimum phase to be $\Phi \simeq 0.1$, the minimum detectable velocity is of the order of $v \simeq 10^{-3} \mathrm{~cm} / \mathrm{s}$. Let us note here that, in principle, the

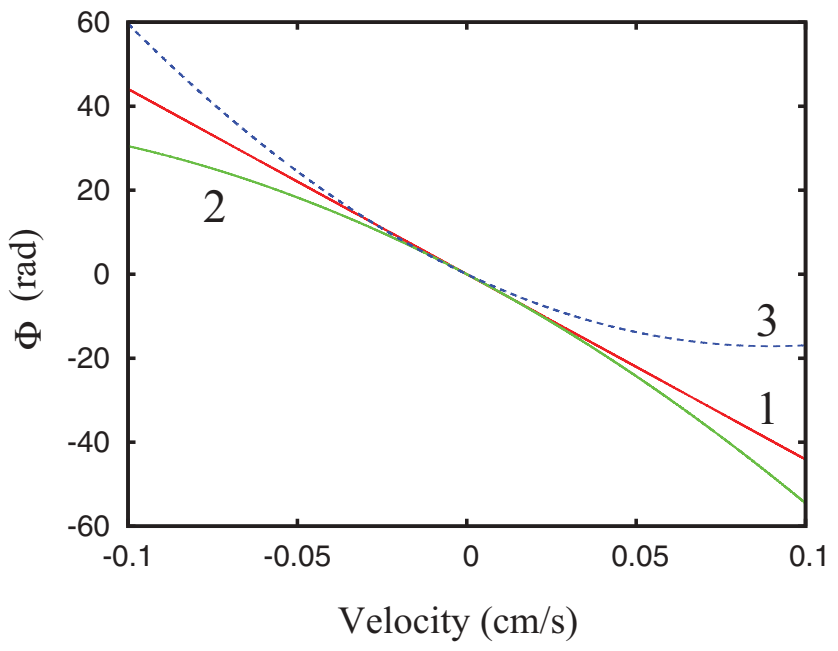

FIG. 5. (Color online) Dependence of the phase shift $\Phi$ on the velocity of the medium for probe detunings equal to (1) $0 \mathrm{~s}^{-1}$, (2) $1.0 \times 10^{3} \mathrm{~s}^{-1}$, and (3) $-2.0 \times 10^{3} \mathrm{~s}^{-1}$.

minimum detectable phase shift is determined by fluctuations of the optical probe field and is given by the signal-to-noise ratio [5],

$$
\delta \Phi_{\min } \simeq \frac{1}{\sqrt{\langle S / N\rangle}},
$$

and can be much smaller.

Using a different method of detection, for example, moving the cell at some frequency (as in Ref. [26]) when the satellites at the frequency of modulation appear near the probe frequency (with intensity $I_{p 0}$ ) due to phase modulation, the intensities of the satellites $I_{ \pm}$are given by

$$
\frac{I_{ \pm}}{I_{p 0}}=\left(\frac{\omega_{a b} L v}{c V_{g}}\right)^{2},
$$

which gives us the same sensitivity to the velocity $v=$ $10^{-3} \mathrm{~cm} / \mathrm{s}$. The frequency of modulation used in the experiment [26] is $10^{4} \mathrm{kHz}$, and the detectable displacement is of the order of $10 \AA$, which is much smaller than the wavelength of laser radiation used in the experiment.

This result shows the accuracy of the position of optical elements that can be controlled by detecting this signal. This technology can be important, for example, for ultrashort (attoseconds, the distance corresponding to 1 as is about $\simeq 3 \AA$ ) physics and microscopy. It enhances the tools that are able to provide control for the positioning of the various elements of the setup.

However, it is worth underscoring here that even with such an enhanced dragging effect due to slow light, one can apply this effect for detection of relative rotation but not for detection of absolute rotation. There is no application to the Sagnac effect $[27,28]$. Indeed, the detectable velocity is of the order of $v \simeq \Omega_{r} R$, the relative velocity due to rotation of the earth $\left(\Omega_{r}\right.$ is the angular velocity of the earth), so, in principle, one might view this technique as promising for detection of rotation (and there were recently some proposals of taking advantage of the ultraslow media for rotation detection [29], but the situation is more complicated). The situation is so subtle that the arguments are worth repeating here because there is 
a great deal of misunderstanding of this issue in the literature (see, for example, [30,31]).

Based upon our calculations above, we can easily estimate the contribution of the cell to the Sagnac effect. The phase velocity of light in the laboratory frame is given by

$$
V_{v}=\frac{\omega}{k}=\frac{\omega_{a b}\left(1+\frac{v}{c}\right)+\delta \omega_{0}\left(1+\frac{v}{V_{g}}\right)}{\frac{\omega_{a b}}{c}\left(1+\frac{v}{c}\right)+\delta \omega_{0}\left(\frac{1}{V_{g}}+\frac{v}{c^{2}}\right)},
$$

which can be rewritten as

$$
V_{v}=\frac{v+\frac{\omega_{a b}+\delta \omega_{0}}{\frac{\omega_{a b}}{c}+\frac{\delta \omega_{0}}{V_{g}}}}{1+\frac{v}{c^{2}} \frac{\omega_{a b}+\delta \omega_{0}}{\frac{\omega_{a b}}{c}+\frac{\delta \omega_{0}}{V_{g}}}},
$$

where

$$
V_{0}=\frac{\omega_{0}}{k_{0}}=\frac{\omega_{a b}+\delta \omega_{0}}{\frac{\omega_{a b}}{c}+\frac{\delta \omega_{0}}{V_{g}}}
$$

is the phase velocity of light in the frame moving with the cell; i.e., the velocity of light in the laboratory frame results from relativistic adding of the phase velocity of light $V_{0}$ and the velocity of frame $v$. Then, we see that when the effect on the phase velocity is large enough, the time difference between light propagating in one direction and in the opposite direction [30] is equal to

$$
\Delta t_{ \pm}=t_{+}-t_{-}=\frac{L}{V_{+v}-v}-\frac{L}{V_{-v}+v}=\frac{4 A_{\mathrm{eff}}}{c^{2}} \Omega_{r},
$$

where $A_{\text {eff }}=L R / 2$ is the effective area for these two cells. The obtained result does not depend on any properties of the slowlight medium and coincides with the well-known travelingtime difference in opposite directions (it is more deeply related to the impossibility of synchronizing clocks along a close line; see Ref. [32]) given by

$$
\Delta t=\frac{4 A}{c^{2}} \Omega
$$

where $A$ is the area of the Sagnac interferometer.

The controversy of the Sagnac effect [28] interpretations and misinterpretations has been nicely reviewed [30,31]. The time difference does not depend on particular properties of waves (slow or fast; linear or nonlinear; electromagnetic radiation, sound waves, or matter waves; we note here that using matter waves [33] allows one to enhance the sensitivity per unit area) used for interferometry. The $c$ in Eq. (33) is a constant of Lorentz transformation, the speed of light in vacuum, and it has no relation to the dispersion of media.

\section{CONCLUSIONS}

In this paper, we theoretically demonstrated a possibility of control of the dragging effect and showed the dependence of the Fizeau effect on higher-order dispersion that can be observed experimentally. It has been shown that the change of phase is sensitive to the motion of the cell with a speed of the order of $10^{-3} \mathrm{~cm} / \mathrm{s}$ and for possible displacements as small as $10 \AA$. Applications of the enhanced dragging effect can be found for efficient modulators of light, position control, and detection of slow motion. Using slow light allows one to detect very slow relative motion of the media and also to develop very efficient phase modulators. The enhanced dragging effect proves efficient coupling between optical fields and mechanical motion, in particular sound waves.

The obtained results can be applied to all cases where the EIT has been achieved. The physics of coherent effects in $\Lambda$-type three-level atoms is related to the excitation of the maximum coherence between two ground states (in alkali atoms, these are the hyperfine levels) under the condition when a special coherent state, the so-called dark state, is formed. EIT has been achieved in atomic [5,6,8] and molecular [34,35] gases, BECs [13], solid-state systems [36-38], metamaterials [39-41], and even the mechanical effect of light [42].

\section{ACKNOWLEDGMENTS}

We thank Eugeniy Mikhailov, Irina Novikova, Andrey Matsko, Kunal Das, and Selim Shahriar for fruitful discussions, comments, and suggestions and gratefully acknowledge the support from the UNT Research Initiation Grant and the summer fellowship UNT program.

\section{APPENDIX: DOPPLER BROADENING}

Doppler broadening of atomic gas can be done in many ways [5,25]. One way is to average the polarization of the medium consisting of moving atoms, namely,

$$
P_{a b, D}\left(\omega_{p}\right)=\int_{-\infty}^{+\infty} d u f(u) P_{a b}\left(\omega_{p}, u\right) .
$$

Then, relating the polarization of the medium to its dielectric function as

$$
P_{a b}=\frac{\epsilon\left(\omega_{p}, u\right)-1}{4 \pi} E_{p}, \quad P_{a b, D}=\frac{\epsilon_{D}\left(\omega_{p}\right)-1}{4 \pi} E_{p},
$$

we can write

$$
\epsilon_{D}=\int_{-\infty}^{+\infty} d u f(u) \epsilon\left(\omega_{p}, u\right),
$$

and for the gas media we obtain Eq. (17). Knowing the index of refraction of the medium, we can directly apply Eq. (9) and obtain the results.

Another way to perform the Doppler broadening is to average the dispersion relation given by Eq. (4). Indeed, for a single-velocity atomic beam, we have

$$
k=\frac{\omega_{p}-k u}{c} n\left(\omega_{p}-k u\right),
$$

where the index of refraction is given by Eq. (16); here $k=k_{p}$. For a beam consisting of many velocity groups, we should add each contribution of each velocity group together as

$k=\int_{-\infty}^{+\infty} d u \frac{\omega_{p}-k u}{c} n\left(\omega_{p}-k u\right) f(u)=\frac{\omega_{p}}{c} n_{D}\left(\omega_{p}\right)$,

where $f(u)$ is the velocity distribution function.

Now, if the atomic gas is moving with velocity $v$, the distribution function is shifted as $f(u-v)$, and

$$
k=\int_{-\infty}^{+\infty} d u \frac{\omega_{p}-k u}{c} n\left(\omega_{p}-k u\right) f(u-v) .
$$


Assuming that the gas velocity $v \ll u_{D}$, we can write

$$
f(u-v) \simeq f(u)-v \frac{d f}{d u}+\frac{v^{2}}{2} \frac{d^{2} f}{d u^{2}} .
$$

Then, Eq. (A6) can be rewritten as

$$
\begin{aligned}
k & =\int_{-\infty}^{+\infty} d u \frac{\omega_{p}-k u}{c} n\left(\omega_{p}-k u\right) f(u-v) \\
& =\int_{-\infty}^{+\infty} d u \frac{\omega_{p}-k u}{c} n\left(\omega_{p}-k u\right)\left(f(u)-v \frac{d f}{d u}+\frac{v^{2}}{2} \frac{d^{2} f}{d u^{2}}\right) \\
& =I_{0}-v I_{1}+\frac{v^{2}}{2} I_{2},
\end{aligned}
$$

where

$$
\begin{aligned}
& I_{0}=\int_{-\infty}^{+\infty} d u \frac{\omega_{p}-k u}{c} n\left(\omega_{p}-k u\right) f(u), \\
& I_{1}=\int_{-\infty}^{+\infty} d u \frac{\omega_{p}-k u}{c} n\left(\omega_{p}-k u\right) \frac{d f}{d u}, \\
& I_{2}=\int_{-\infty}^{+\infty} d u \frac{\omega_{p}-k u}{c} n\left(\omega_{p}-k u\right) \frac{d^{2} f}{d u^{2}} .
\end{aligned}
$$

Then, similar to Eq. (A5),

$$
I_{0}=\frac{\omega_{p}}{c} n_{D}\left(\omega_{p}\right)
$$

Performing integration by parts, we obtain

$$
\begin{aligned}
I_{1} & =\int_{-\infty}^{+\infty} d u \frac{\omega_{p}-k u}{c} n\left(\omega_{p}-k u\right) \frac{d f}{d u} \\
& =k \int_{-\infty}^{+\infty} d u f(u) \frac{d}{d \omega_{p}}\left(\frac{\omega_{p}-k u}{c} n\left(\omega_{p}-k u\right)\right) \\
& =\frac{k}{c} \frac{d}{d \omega_{p}}\left[\omega_{p} n_{D}\left(\omega_{p}\right)\right], \\
I_{2} & =\frac{k^{2}}{c} \frac{d^{2}}{d \omega_{p}^{2}}\left[\omega_{p} n_{D}\left(\omega_{p}\right)\right] .
\end{aligned}
$$

Finally, we obtain

$$
\begin{aligned}
k= & \frac{\omega_{p}}{c} n_{D}\left(\omega_{p}\right)-\frac{k v}{c} \frac{d}{d \omega_{p}}\left[\omega_{p} n_{D}\left(\omega_{p}\right)\right] \\
& +\frac{k^{2} v^{2}}{2 c} \frac{d^{2}}{d \omega_{p}^{2}}\left[\omega_{p} n_{D}\left(\omega_{p}\right)\right],
\end{aligned}
$$

which coincides with Eq. (5), from which Eq. (9) follows.
[1] H. Fizeau, Ann. Chim. Phys. 57, 385 (1859); C. R. Acad. Sci. (Paris) 33, 349 (1851); A. Fresnel, Ann. Chim. Phys. 9, 57 (1818).

[2] I. Lerche, Am. J. Phys. 45, 1154 (1977).

[3] L. D. Landau and E. M. Lifshitz, Electrodynamics of Continious Media, Course of Theoretical Physics, Vol. 8 (Pergamon, Oxford, 1960).

[4] E. Arimondo, Prog. Opt. 35, 257 (1996).

[5] M. O. Scully and M. S. Zubairy, Quantum Optics (Cambridge University Press, Cambridge, 1997).

[6] S. E. Harris, Phys. Today 50(7), 36 (1997).

[7] S. E. Harris, Phys. Rev. Lett. 70, 552 (1993).

[8] M. Fleischhauer, A. Imamoglu, and J. P. Marangos, Rev. Mod. Phys. 77, 633 (2005).

[9] O. Kocharovskaya and Ya. I. Khanin, Sov. Phys. JETP 63, 945 (1986).

[10] V. A. Sautenkov, Y. V. Rostovtsev, C. Y. Ye, G. R. Welch, O. Kocharovskaya, and M. O. Scully, Phys. Rev. A 71, 063804 (2005).

[11] V. A. Sautenkov, H. Li, Y. V. Rostovtsev, and M. O. Scully, Phys. Rev. A 81, 063824 (2010).

[12] I. Carusotto, M. Artoni, G. C. La Rocca, and F. Bassani, Phys. Rev. A 68, 063819 (2003).

[13] L. V. Hau, S. E. Harris, Z. Dutton, and C. H. Behroozi, Nature (London) 397, 594 (1999); C. Liu, Z. Dutton, C. H. Behroozi, and L. V. Hau, ibid. 409, 490 (2001).

[14] M. M. Kash, V. A. Sautenkov, A. S. Zibrov, L. Hollberg, G. R. Welch, M. D. Lukin, Y. Rostovtsev, E. S. Fry, and M. O. Scully, Phys. Rev. Lett. 82, 5229 (1999); D. Budker, D. F. Kimball, S. M. Rochester, and V. V. Yashchuk, ibid. 83, 1767 (1999).

[15] L. J. Wang, A. Kuzmich, and A. Dogariu, Nature (London) 406, 277 (2000); A. Dogariu, A. Kuzmich, and L. J. Wang, Phys. Rev. A 63, 053806 (2001).
[16] G. S. Agarwal, T. N. Dey, and S. Menon, Phys. Rev. A 64, 053809 (2001).

[17] E. E. Mikhailov, V. A. Sautenkov, Y. V. Rostovtsev, and G. R. Welch, J. Opt. Soc. Am. B 21, 425 (2004); Q. Sun, Y. V. Rostovtsev, J. P. Dowling, M. O. Scully, and M. S. Zubairy, Phys. Rev. A 72, 031802 (2005).

[18] A. B. Matsko, Y. V. Rostovtsev, M. Fleischhauer, and M. O. Scully, Phys. Rev. Lett. 86, 2006 (2001).

[19] Y. V. Rostovtsev, Z.-E. Sariyanni, and M. O. Scully, Phys. Rev. Lett. 97, 113001 (2006).

[20] V. A. Sautenkov, Y. V. Rostovtsev, and M. O. Scully, Phys. Rev. A 72, 065801 (2005).

[21] M. Fleischhauer and M. D. Lukin, Phys. Rev. Lett. 84, 5094 (2000); Phys. Rev. A 65, 022314 (2002); D. F. Phillips, A. Fleischhauer, A. Mair, R. L. Walsworth, and M. D. Lukin, ibid. 86, 783 (2001).

[22] A. B. Matsko, Y. V. Rostovtsev, O. Kocharovskaya, A. S. Zibrov, and M. O. Scully, Phys. Rev. A 64, 043809 (2001); C. Mewes and M. Fleischhauer, ibid. 66, 033820 (2002); T. N. Dey and G. S. Agarwal, ibid. 67, 033813 (2003).

[23] O. Kocharovskaya, Y. Rostovtsev, and M. O. Scully, Phys. Rev. Lett. 86, 628 (2001).

[24] M. S. Shahriar, G. S. Pati, R. Tripathi, V. Gopal, M. Messall, and K. Salit, Phys. Rev. A 75, 053807 (2007).

[25] Y. Rostovtsev, I. Protsenko, H. Lee, and A. Javan, J. Mod. Opt. 49, 2501 (2002); H. Lee, Y. Rostovtsev, C. J. Bednar, and A. Javan, Appl. Phys. B 76, 33 (2003); A. B. Matsko, D. V. Strekalov, and L. Maleki, Opt. Express 13, 2210 (2005).

[26] A. Zhang, V. A. Sautenkov, Y. V. Rostovtsev, and G. R. Welch, Proc. SPIE 5735, 138 (2005).

[27] M. G. Sagnac, C. R. Acad. Sci. 157, 708 (1913).

[28] E. J. Post, Rev. Mod. Phys. 39, 475 (1967). 
[29] U. Leonhardt and P. Piwnicki, Phys. Rev. A 62, 055801 (2000). [30] G. B. Malykin, Phys. Usp. 43, 1229 (2000).

[31] P. G. Eleseev, Opto-Electron. Rev. 16, 118 (2008).

[32] L. D. Landau and E. M. Lifshitz, The Classical Theory of Fields, 4th ed., Course of Theoretical Physics, Vol. 2 (Pergamon, Oxford, 1971).

[33] F. Zimmer and M. Fleischhauer, Phys. Rev. Lett. 92, 253201 (2004).

[34] J. B. Qi and A. M. Lyyra, Phys. Rev. A 73, 043810 (2006); L. Li, P. Qi, A. Lazoudis, E. Ahmed, and A. M. Lyyra, Chem. Phys. Lett. 403, 262 (2005).

[35] H. Li, H. Chen, M. A. Gubin, Y. V. Rostovtsev, V. A. Sautenkova, and M. O. Scully, Laser Phys. 20, 1725 (2011).

[36] K. Ichimura, K. Yamamoto, and N. Gemma, Phys. Rev. A 58, 4116 (1998).
[37] Y. Zhao, C. Wu, B. S. Ham, M. K. Kim, and E. Awad, Phys. Rev. Lett. 79, 641 (1997).

[38] B. S. Ham, M. S. Shahriar, and P. R. Hemmer, Opt. Lett. 22, 1138 (1997).

[39] N. Liu, T. Weiss, M. Mesch, L. Langguth, U. Eigenthaler, M. Hirscher, C. Snnichsen, and H. Giessen, Nano Lett. 10, 1103 (2010).

[40] V. A. Fedotov, M. Rose, S. L. Prosvirnin, N. Papasimakis, and N. I. Zheludev, Phys. Rev. Lett. 99, 147401 (2007); N. Papasimakis, V. A. Fedotov, N. I. Zheludev, and S. L. Prosvirnin, ibid. 101, 253903 (2008).

[41] S. Y. Chiam, R. Singh, C. Rockstuhl, F. Lederer, W. Zhang, and A. A. Bettiol, Phys. Rev. B 80, 153103 (2009).

[42] G. S. Agarwal and S. Huang, Phys. Rev. A 81, 041803 (2010). 